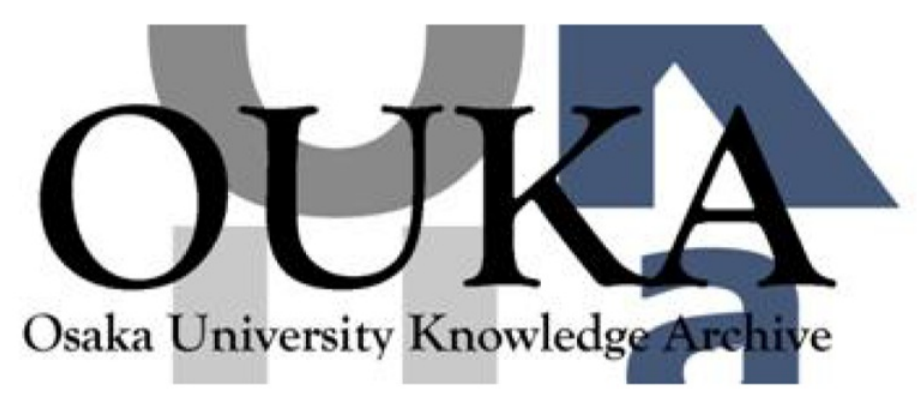

\begin{tabular}{|c|l|}
\hline Title & $\begin{array}{l}\text { Mechanisms of ultrasonically induced } \\
\text { fibrillation of amyloid } \beta_{1-40} \text { peptides }\end{array}$ \\
\hline Author(s) & $\begin{array}{l}\text { Uesugi, Kentaro; Ogi, Hirotsugu; Fukushima, } \\
\text { Masahiko et al. }\end{array}$ \\
\hline Citation & $\begin{array}{l}\text { Japanese Journal of Applied Physics. 52 } \\
\text { p.07HE10 }\end{array}$ \\
\hline Issue Date & $2013-06-20$ \\
\hline oaire:version & AM \\
\hline URL & https://hdl. handle. net/11094/84169 \\
\hline rights & \\
\hline Note & \\
\hline
\end{tabular}

Osaka University Knowledge Archive : OUKA

https://ir. Library. osaka-u. ac. jp/

Osaka University 


\title{
Mechanisms of Ultrasonically Induced Fibrillation of Amyloid $\beta_{1-40}$ Peptides
}

\author{
Kentaro Uesugi ${ }^{1}$, Hirotsugu Ogi ${ }^{*}$, Masahiko Fukushima ${ }^{1}$, Masatomo $\mathrm{So}^{2}$, Hisashi \\ YAGI $^{2}$, Yuji Goto ${ }^{2}$, and Masahiko HiRAo ${ }^{1}$ \\ ${ }^{1}$ Graduate School of Engineering Science, Osaka University, Toyonaka, Osaka 560-8531, Japan \\ ${ }^{2}$ Institute for Protein Research, Osaka University, Suita, Osaka 565-0871, Japan
}

We systematically study the relationship between the ultrasonically induced aggregation behavior of amyloid $\beta_{1-40}$ peptide and acoustic pressures to clarify the dominant mechanism of the aggregation. With ultrasonic irradiation, the thioflavin- $\mathrm{T}$ (ThT) level of the $\mathrm{A} \beta$ solution rises after a lag time, takes a maximum at $\sim 5 \mathrm{~h}$, and remains unchanged or decreases. Thus, we monitor the ThT level at $5 \mathrm{~h}$ to evaluate the progress of the $\beta$-sheet structure and investigate its correlation with the acoustic pressures of fundamental and harmonics waves. The second-harmonics-wave amplitude shows the highest correlation with the ThT level, indicating the dominant contribution of cavitation bubbles to the fibrillation phenomenon. The influence of solution $\mathrm{pH}$ and $\mathrm{Ar}$ gas are investigated to identify the aggregation mechanism. As a result, local condensation of the peptide due to the high affinity of hydrophobic residues to the bubble-solution interface causes a highly supersaturated solution, leading to precipitation of $\beta$-sheet-rich nuclei.

\section{Introduction}

Alzheimer's disease (AD) is known as a major cause of dementia. ${ }^{1)}$ It is widely recognized that the pathogenesis of $\mathrm{AD}$ is deeply related to the aggregation behavior of amyloid $\beta(\mathrm{A} \beta$ ) peptides. ${ }^{2-4)}$ However, the pathogenic mechanism still remains unclear. A $\beta$ is one of amyloidosis proteins such as $\beta_{2}$-microglobulin $(\beta 2-\mathrm{m}), \alpha$-synuclein, and prion protein $(\operatorname{PrP})$, which form amyloid fibrils through intermediate aggregates and their transition to nuclei. ${ }^{5-9)}$ The common characteristics of amyloid fibrils are that they have highly regular cross- $\beta$ structures where $\beta$-strands align perpendicularly to the fibril longitudinal axis. ${ }^{10)}$

Normally, the onset age of $\mathrm{AD}$ is over the 60 . Hence, $\mathrm{A} \beta$ peptides need a long time to form aggregations in vivo: the energy barrier for the structural change from monomer to nucleus is generally very high. This characteristic has prevented us from clarifying the pathogenic mechanism of AD. Therefore, it is highly important to develop a methodology to accelerate aggregation of the peptides.

Agitation procedures by stirring, shaking, and rotating solutions have been adopted to accelerate the aggregation of proteins. ${ }^{11)}$ Goto and coworkers ${ }^{7,12,13)}$ have recently displayed

*E-mail:ogi@me.es.osaka-u.ac.jp 
that ultrasonic irradiation (UI) significantly induced the formation of amyloid fibrils and produced highly homogeneous fibrils for $\beta 2-\mathrm{m}$. This phenomenon has been reported for other

amyloidosis proteins as well, including $\alpha$-synuclein ${ }^{8)}$ and $\operatorname{PrP}{ }^{9)}$ Therefore, the UI method is expected to be efficient for the acceleration of the aggregation of $\mathrm{A} \beta$ peptides, although no systematic study has been carried out.

UI of aqueous solutions produces cavitation bubbles: ultrasonic waves cause nearly adiabatic collapse of the bubbles, which generates microscopic reaction fields of high temperature and high pressure, and also produces free radical species in the solution. These extreme reaction fields allow the decomposition of various organic compounds. ${ }^{14-17)}$ Besides, UI has been used to break preformed amyloid fibrils to smaller seeds. ${ }^{18,19)}$ However, ultrasonically induced aggregation $^{7-9,12,13)}$ means an enhancement of polymerization, which is a reverse trend to the previous sonochemical reactions. Therefore, this mechanism remains unclear. Clarification of the mechanism is important to control the structure and acceleration rate of aggregates, which will contribute to the drug discovery for AD.

Recently, it has been indicated that the lag time for the nucleation of a PrP aggregate is dependent on the ultrasonic power, ${ }^{9)}$ which was determined using calorimetry and potassium iodide oxidation methods. ${ }^{20)}$ However, this method fails to identify the dominant nucleation mechanism because the ultrasonic power is related to almost all acoustic parameters. Considering that the cavitation bubble plays a central role in sonochemical reactions, ${ }^{21-23)}$ it is necessary to investigate the dependence of the aggregation phenomena on the acoustic pressures of fundamental and harmonics-wave modes individually.

Here, we systematically study the ultrasonically induced fibrillation behavior for A $\beta$ peptides and investigate the relationships between the aggregation degree and acoustic pressures of fundamental and harmonics waves. These correlations are studied by changing the $\mathrm{pH}$ of the solution and also by replacing the dissolved gas with Ar gas to evaluate the influence of the protein charge and the hot-spot temperature at the bubble collapse, respectively.

\section{Possible Effects of Ultrasonic Irradiation}

We suggest three possible dominant effects caused by UI. First is the acceleration of agitation: when a solution is irradiated with an ultrasonic wave, peptides move over a distance longer than their Brownian distance, and they self-assemble more frequently to cause nucleation. The contribution of this effect to the protein aggregation can be evaluated by investigating the dependences of the aggregated degree on the amplitude and frequency of the fundamental wave.

Second is the local condensation of the peptides around cavitation bubbles. The hydrophobic residues tend to be attracted to the bubble surface, and the peptides assemble along the surface so as to expose their hydrophilic residues toward the solution side. Also, when the peptides are positively charged, they are electrically attracted to the bubble surface because 
Jpn. J. Appl. Phys.

Regular Paper

the bubble surface is generally negatively charged. ${ }^{24,25)}$ Because the bubble collapse occurs in a short time, the concentration of the peptide increases, locally producing supersaturated regions and then nucleation of aggregates. We can evaluate this effect by investigating the relationship between the aggregated degree and the acoustic pressures of harmonics at different $\mathrm{pHs}$ of the solution across the isoelectric point of the peptides.

Third is the reaction promotion by the temperature increase at the bubble collapse. We can evaluate this contribution by investigating the frequency dependence of aggregation and the use of Ar-gas saturated solution.

\section{Measurements}

\subsection{Materials and instruments}

$\mathrm{A} \beta_{1-40}$ peptides were used throughout this study. Lyophilized $\mathrm{A} \beta_{1-40}$ peptides were purchased from the Peptide Institute (product No. 4307-v). Phosphate buffered saline (PBS) powder, acetate buffered saline (ABS), dimethyl sulfoxide (DMSO), and thioflavin T (ThT) were purchased from Wako Pure Chemical Industries.

We used two $200 \mathrm{~W}$ continuous-wave ultrasonic irradiation systems KAIJO 43013 and 4021 for 26 and $200 \mathrm{kHz}$, respectively. The atomic-force-microscopy (AFM) system was a Shimadzu SPM-9600. ThT fluorescence was measured using a fluorescence spectrometer JASCO FP6200. We used a needle-type hydrophone Toray Engineering NH8193 for calibration of the acoustic pressure.

\subsection{Ultrasonic irradiation experiments}

Figure 1 shows the experimental setup. The ultrasonic transducer was located in a water bath, where the temperature was kept at $\sim 15{ }^{\circ} \mathrm{C}$ by circulating cold water. Ten microtubes (polypropylene and $1.7 \mathrm{ml}$ vol) including $500 \mu \mathrm{l}$ solution were set in the tube holder, and they were located above the transducer.

The ultrasonic waveform was acquired for $30 \mathrm{~s}$ in each microtube using a 1-mm-diameter custom-built PZT probe. Fast Fourier transform (FFT) was performed on the acquired waveform to extract the detected voltages of fundamental and higher harmonics waves using each peak value, which was converted into pressure values after calibration using the needle-type hydrophone. With the measurement setup in Fig. 1, we can vary the acoustic intensity in different microtubes even with the same driving power by a factor of 10 .

The lyophilized $\mathrm{A} \beta_{1-40}$ was dissolved in DMSO with stirring at $200 \mathrm{rpm}$ for $5 \mathrm{~min}$, and diluted by $\mathrm{ABS}(\mathrm{pH} 4.6)$ or $\mathrm{PBS}(\mathrm{pH} 7.4)$ including $0.2 \mathrm{M} \mathrm{NaCl}$ to obtain a final peptide concentration of $100 \mu \mathrm{g} / \mathrm{ml}$. (DMSO:buffer solution=1:4.)

We used two types of ultrasonic transducer with fundamental frequencies of 26 and 200 kHz. A $500 \mu \mathrm{l} \mathrm{A} \beta$ solution was poured to each microtube, and UI was performed. A single UI sequence consisted of $1 \mathrm{~min}$ irradiation and $9 \mathrm{~min}$ incubation. This $10 \mathrm{~min}$ sequence was 
repeated.

To investigate the effect of dissolved gas in the $\mathrm{A} \beta$ solution on fibrillation, we used an Ar-saturated solution: Bubbling was carried out in PBS with Ar gas for 30 min to replace the dissolved gas (air) with Ar. The $\mathrm{A} \beta$ solution was prepared using this Ar-saturated buffer solution. Furthermore, we replaced air inside the microtube with Ar gas and shielded it with a cap to prevent replacement of the dissolved Ar gas with air by ultrasonic irradiation.

\subsection{ThT fluorescence assay}

ThT selectively binds to the $\beta$-sheet structures and produces intense light emission, ${ }^{26,27)}$ and it has been widely adopted to evaluate the formation of protofibrils and their extension. ${ }^{12,13,28-30)}$ We prepared a stock solution by dissolving powdered ThT into a $50 \mathrm{mM}$ glycine/sodium-hydroxide buffer solution ( $\mathrm{pH} 8.5$ ) to obtain a final concentration of $5 \mu \mathrm{M}$. The solution was wrapped in aluminum foil and stored at $4{ }^{\circ} \mathrm{C}$ before use. A $5 \mu \mathrm{l} A \beta$ solution was mixed with a 50- $\mu \mathrm{l}$ ThT solution in a quartz-glass cell for the fluorospectrophotometer. The fluorescence intensity was measured at $485 \mathrm{~nm}$ for the excitation at $450 \mathrm{~nm}$. Because all the ThT assays were performed after ultrasonication, no direct influence of ultrasonication on ThT ability is involved.

\subsection{AFM observation}

We used AFM to observe the morphologies of the ultrasonicated A $\beta$ peptides. A $10 \mu \mathrm{l}$ ultrasonicated $\mathrm{A} \beta$ solution was dropped onto a freshly cleaved mica plate, dried for 5 min, rinsed by ultrapure water, and dried to make the substances in the solution attach to the mica plate. The tapping-mode measurement was adopted with a silicon cantilever, showing the resonance frequency near $300 \mathrm{kHz}$.

\section{Results}

\subsection{Measurement of acoustic pressure}

Figure 2 shows parts of acquired waveforms and corresponding FFT spectra in four different microtubes when the $26 \mathrm{kHz}$ ultrasonic transducer was used. The FFT peak values of harmonics, subharmonics, and ultraharmonics become higher as the driving power to the transducer increases.

\subsection{Acceleration of aggregation for $A \beta$ peptides by UI}

Because the acceleration of aggregation of $\mathrm{A} \beta$ peptides by UI has never been reported, we first investigated this using the $26 \mathrm{kHz}$ transducer with the $\mathrm{A} \beta$ solution at $\mathrm{pH} 4.6$ and compared the result with those when the solution was stirred at 1,200 rpm and incubated at room temperature. The results are shown in Fig. 3. The ThT level remained unchanged with the incubation treatment for $120 \mathrm{~h}$, and it was slightly increased at $3 \mathrm{~h}$ but was saturated at 
Jpn. J. Appl. Phys.

Regular Paper

$24 \mathrm{~h}$ with the stirring treatment. Thus, the aggregation ability of $\mathrm{A} \beta_{1-40}$ is not high. However, it increased with UI after a lag time of $\sim 2 \mathrm{~h}$ to a level, that would never have been achieved by the stirring and incubation treatments. This result confirms that UI displays a significant ability to accelerate the aggregation reaction of $\mathrm{A} \beta$ peptides as well as $\beta 2-\mathrm{m}^{12)}$ and $\mathrm{PrP} .{ }^{9)}$

Next, we investigated the relationship between the evolution of the ThT level and the acoustic pressure of the second harmonics. The representative results are shown in Fig. 4. To evaluate the lag time and the saturated value, we fitted the sigmoid function

$$
f(t)=y_{0}+\frac{y_{\max }}{1+\exp \left(\frac{t_{0.5}-t}{\tau}\right)}
$$

to the measured ThT level. Here, $y_{0}$ and $y_{\max }$ are the initial and maximum fluorescence intensities, respectively, $t_{0.5}$ is the time at which the fluorescence intensity reaches one-half of the maximum value, and $\tau$ is the aggregation rate constant. ${ }^{31)}$ The time course of the ThT level is explained by the sigmoid function, and the maximum ThT level increased and the lag time apparently decreased as the acoustic pressure increased. However, the ThT level gradually decreased after its maximum at $5 \mathrm{~h}$ when the acoustic pressure is large.

\subsection{Acoustic-pressure dependences of ThT level}

To identify the dominant cause of ultrasonically induced aggregation, we systematically investigated the acoustic-pressure dependences of the ThT level at $5 \mathrm{~h}$ for two driving frequencies (26 and $200 \mathrm{kHz}$ ), two $\mathrm{pH}$ environments ( $\mathrm{pH} 4.6$ and 7.4), and with and without Ar gas. The results are shown in Fig. 5. It should be noted that the acoustic pressure of the fundamental wave fails to show a good correlation with the ThT level, whereas those of harmonics show higher correlations with the ThT value. In particular, the correlation of second harmonics with the ThT level is the highest. Furthermore, no significant difference was observed for the ThT levels between solutions at $\mathrm{pH} 4.6$ and 7.4. The ThT level in the argon-gas saturated solution slightly rises.

\subsection{Morphologies of aggregates}

Interestingly, $\mathrm{A} \beta$ aggregates produced by UI at different $\mathrm{pHs}$ of the solution showed different morphologies. At $\mathrm{pH} 4.6$, small aggregates with $\sim 200 \mathrm{~nm}$ diameter were dominant, as shown in Fig. 6(a), whereas at pH 7.4, short fibrils of $\sim 1 \mu \mathrm{m}$ length, as shown in Fig. 6(b), and aggregates larger than $5 \mu \mathrm{m}$, as shown in Fig. 6(c), consisting of gathered short fibrils were observed. However, long fibrils were few and they were not dominant structures at both pHs.

\section{Discussion}

\subsection{Time course of ThT level during UI}

Figure 3 clearly demonstrates that UI causes acceleration of fibrillation much more efficiently than the high-speed stirring procedure at 1,200 rpm and room-temperature incubation. 
Jpn. J. Appl. Phys.

Regular Paper

The ThT level during UI application is maximized at $5 \mathrm{~h}$ and then decreases when the acoustic pressure is high (solid squares in Fig. 4). Such a decrease in the ThT level after the maximum was also reported for $\beta 2-\mathrm{m},{ }^{12,13)}$ and it was suggested that ThT fluorescence activity would have been suppressed by UI, because previous studies included ThT in the $\beta 2$-m solution from the start. However, we applied UI to the ThT-free A $\beta$ solution and then added ThT in the solution in the ThT fluorescence assay. Therefore, this study confirms that the lowering of the ThT level by UI is not related to the degradation of ThT activity. It is therefore reasonable to attribute the decrease to the fragmentation of amyloid fibrils and breakage of $\beta$-sheet structures by UI. The AFM images show that the representative length of a fibril produced by UI is about $1 \mu \mathrm{m}$, as shown in Fig. 6 . Thus, it is expected that the equilibrium length is determined by the balance between elongation and fragmentation of the fibrils in the case of UI-induced aggregation.

\subsection{Relationship between acoustic pressure and fibrillation}

Because the maximum ThT value appears at near $5 \mathrm{~h}$, it is possible to evaluate the influence of acoustic pressure on the aggregation degree and the lag time efficiently by monitoring the ThT level at $5 \mathrm{~h}$. We thus used this $5 \mathrm{~h}$ ThT value for the systematic study of the ultrasonically induced aggregation of the $\mathrm{A} \beta$ peptide.

Clarification of the ultrasonically induced aggregation mechanism requires identification of a dominant effect among the three ultrasonic effects described in sect. 2: (I)simple enhancement of agitation, (II) local condensation of peptide, and (III) temperature increase near the bubble. As shown in Fig. 5, the correlation of the fundamental acoustic pressure with the ThT level is poor, and it is poorer at $200 \mathrm{kHz}$ than at $26 \mathrm{kHz}$. Therefore, the effect (I) cannot be dominant for the fibrillation, because the fundamental mode has a large amplitude to carry the peptide ove a long distance, and a higher frequency enhances the agitation effect more effectively. Thus, this result indicates that measuring the acoustic pressure of the fundamental wave will fail to clarify the aggregation mechanism.

Cavitations are generated when an aqueous solution is irradiated with a high-intensity ultrasonic wave, and they cause strong nonlinearity in the acoustic pressure through shock waves at the bubble collapse and highly nonlinear bubble dynamics. ${ }^{32-34)}$ Therefore, the result in Fig. 5, showing higher correlations between the acoustic pressures of harmonics and the ThT level, strongly indicates that the ultrasonically induced aggregation phenomenon deeply involves the cavitation phenomenon. Thus, we consider effect (II). Hydrophobic amino acid residues of the peptides are attracted to the gas-liquid interface of cavitation bubbles, exposing their hydrophilic residues to the solution side. Therefore, the peptides assemble along the bubble surface accompanied by the bubble growth, and local condensation is achieved by subsequent instantaneous collapse of the bubble. For example, Iefuji et al. reported that crystallization of the protein was accelerated by applying an intense femtosecond light pulse, ${ }^{35}$ ) 
Jpn. J. Appl. Phys.

Regular Paper

which causes bubble growth and collapse due to local boiling with the laser beam, leading to nucleation through local condensation. It is expected that the effect will become noticeable for low frequencies because the bubble radius and the surface area increase as the frequency decreases. We simulated the dynamics of a single bubble in water using the Keller-Miksis equation: ${ }^{36-38)}$

$$
\begin{gathered}
\left(1-\frac{\dot{R}}{c_{\infty}}\right) R \ddot{R}+\frac{3}{2}\left(1-\frac{1}{3} \frac{\dot{R}}{c_{\infty}}\right) \dot{R}^{2}= \\
\left(1+\frac{\dot{R}}{c_{\infty}}\right) \frac{1}{\rho_{\infty}}\left\{p_{b}-P_{A} \sin \left[\omega\left(t+\frac{r}{c_{\infty}}\right)\right]-p_{\infty}\right\}+\frac{R}{\rho_{\infty} c_{\infty}} \frac{\partial p_{b}}{\partial t}, \\
p_{b}=\left(p_{\infty}+\frac{2 \sigma}{R_{0}}\right)\left(\frac{R_{0}}{R}\right)^{3 \gamma}-\frac{2 \sigma}{R}-\frac{4 \mu \dot{R}}{R} .
\end{gathered}
$$

Here, $R, c_{\infty}, \rho_{\infty}, p_{\infty}, \mu, \sigma$, and $\gamma$ denote the bubble radius, acoustic velocity in the liquid, density of the liquid, hydrostatic pressure, viscosity of the liquid, surface tension of bubble, and ratio of specific heat, respectively. $P_{A}$ is the driving-acoustic-pressure amplitude and $R_{0}$ is the normal radius. The result in Fig. 7 (a) shows that the maximum bubble radius at 26 $\mathrm{kHz}$ is larger than that at $200 \mathrm{kHz}$ by a factor of 3.5. In fact, the ThT level at $26 \mathrm{kHz}$ is considerably higher, supporting the dominant contribution of effect (II).

The bubble is known to be negatively charged, ${ }^{24,25)}$ and positively charged proteins could be electrically collected near the bubble surface. The isoelectric point of the $\mathrm{A} \beta$ peptide occurs at $\mathrm{pH} 5.2$, and it should be positively or negatively charged at $\mathrm{pH} 4.6$ or 7.4 , respectively. If the electrical interaction were dominant, we would expect an insignificant increase in the ThT level at $\mathrm{pH} 7.4$ because of repulsive interaction between the peptides and bubbles. However, the ThT level at $\mathrm{pH} 7.4$ is comparable to or slightly higher than that at $\mathrm{pH} 4.6$ in Fig. 5, indicating that this electrical interaction is negligible.

Effect (III) also appears to affect the fibrillation. The solubility of proteins often decreases as temperature increases, which is dependent on the characteristics of hydrophobic residues. ${ }^{39)}$ This effect will enhance the supersaturation degree. Besides, the reaction rate for fibrillation will be increased at elevated temperatures. It is known that the temperature at the bubble collapse becomes higher by replacing the dissolved gas with Ar because of its larger specificheat ratio. As shown in the simulation in Fig. 7(b), the maximum temperature at bubble collapse in an air-saturated solution $(19,045 \mathrm{~K})$ increases in an Ar-saturated solution (36,805 $\mathrm{K}$ ) by a factor of 1.93. Thus, the replacement of the dissolved gas with Ar enhances sonochemical reaction rates. For example, the decomposition reaction of methylene blue by UI is accelerated in the Ar-gas-saturated solution. ${ }^{14)}$ UI with Ar gas appears to increase the ThT level slightly (inverted triangles in Fig. 5), indicating that the high temperature region around bubbles contributes to fibrillation, although this effect is too local to drastically accelerate the aggregation. 
Jpn. J. Appl. Phys.

Regular Paper

Therefore, the most important factor for the ultrasonically induced aggregation phenomenon of $\mathrm{A} \beta$ peptides is local condensation near bubbles through adsorption of their hydrophobic residues on the bubble surface. Yoshimura et al. proposed a precipitation model of $\beta 2-\mathrm{m}$ by UI, where nucleation is caused by the transition to the labile region from the metastable region in the phase diagram. ${ }^{29)} \mathrm{A}$ similar phenomenon will apply to $\mathrm{A} \beta$ peptides: UI causes local condensation of the peptide, where the amount of dissolution of the peptide increases and the degree of supersaturation is enhanced. Thus, the transition from the metastable region to the labile region is conducted, as indicated by the arrow in Fig. 8(a). The temperature near the bubble increases after collapse, backing up this transition as well. In other words, an enhanced supersaturation state accompanies the change in the free-energy landscape, because the intermediate state should become more stabilized, ${ }^{40)}$ corresponding to the lowering of the apparent energy barrier for the nucleation from the monomer. This concept is illustrated in Fig. 8(b). Once the nucleus appears, fibrillation progresses naturally.

\section{Conclusions}

We confirmed the promotion of fibrillation of $\mathrm{A} \beta_{1-40}$ peptides by ultrasonic irradiation using ThT fluorescence assay. The ThT level increased after a lag time, took a maximum at 5 $\mathrm{h}$, and decreased when the second harmonics amplitude was large. We monitored the $5 \mathrm{~h}$ ThT level and systematically studied correlations between the acoustic pressures of fundamental and harmonics with the ThT level. Notable results are (i) the acoustic pressure of the second harmonics wave exhibits the highest correlation, (ii) the ThT level is higher with the $26 \mathrm{kHz}$ transducer than with the $200 \mathrm{kHz}$ transducer even with the same acoustic pressure, (iii) no significant $\mathrm{pH}$ dependence is observed even across the isoelectrical point, (iv) a slight increase in the ThT level appeared in UI for the Ar-gas-saturated solution. From these results, we conclude that the dominant factor for ultrasonically induced fibrillation of the peptide is the local condensation of the peptide and the temperature increase near cavitation bubbles, which enhances the degree of supersaturation and lowers the energy barrier for nucleation.

\section{Acknowledgement}

This study was supported by the Funding Program for Next Generation World-Leading Researchers by the Cabinet Office, Government of Japan. 
Jpn. J. Appl. Phys.

Regular Paper

\section{References}

1) M. M. B. Breteler, J. J. Claus, C. M. Duijn, L. J. Launer, and A. Hofman: Epidemiol. Rev. 14 (1992) 59.

2) D. J. Selkoe: Neuron 6 (1991) 487.

3) J. A. Hardy and G. A. Higgins: Science 256 (1992) 184.

4) J. A. Hardy and D. J. Selkoe: Science 297 (2002) 353.

5) G. Bitan, M. D. Kirkitadze, A. Lomakin, S. S. Vollers, G. B. Benedek, and D. B. Teplow: Proc. Natl. Acad. Sci. U. S. A. 100 (2003) 330.

6) N. Benseny-Cases, M. Cocera, and J. Cladera: Biochem. Biophys. Res. Commun. 361 (2007) 916.

7) Y. Ohhashi, M. Kihara, H. Naiki, and Y. Goto: J. Mol. Biol. 280 (2005) 32843.

8) K. H. Jin, E. Chatani, Y. Goto, and S. R. Paik: J. Microbiol. Biotechnol. 17 (2007) 2027.

9) K. Yamaguchi, T. Matsumoto, and K. Kuwata: Protein Sci. 21 (2012) 38.

10) T. Lührs, C. Ritter, M. Adrian, D. Riek-Loher, B. Bohrmann, H. Döbeli, D. Schubert, and R. Riek: Proc. Natl. Acad. Sci. U. S. A. 102 (2005) 17342.

11) L. Giehm and D. E. Otzen: Anal. Biochem. 400 (2010) 270.

12) E. Chatani, Y. Lee, H. Yagi, Y. Yoshimura, H. Naiki, and Y. Goto: Proc. Natl. Acad. Sci. U. S. A. 106 (2009) 11119.

13) M. So, H. Yagi, K. Sakurai, H. Ogi, H. Naiki, and Y. Goto: J. Mol. Biol. 412 (2011) 568.

14) H. Ogi, Y. Tomiyama, Y. Shoji, T. Mizugaki, and M. Hirao: Jpn. J. Appl. Phys. 45 (2006) 4678.

15) J. Dewulf, H. V. Langenhove, A. D. Visscher, and S. Sabbe: Ultrason. Sonochem. 8 (2001) 4678.

16) J. R. Thomas: J. Phys. Chem. 63 (1959) 1725.

17) B. Park, D. Shin, E. Cho, and J. Khim: Jpn. J. Appl. Phys. 51 (2012) 07GD07.

18) A. K. Paravastu, I. Qahwash, R. D. Leapman, S. C. Meredith, and R. Tycko: Proc. Natl. Acad. Sci. U. S. A. 106 (2009) 7443.

19) K. Pauwels, T. L. Williams, K. L. Morris, W. Jonckheere, A. Vandersteen, G. Kelly, J. Schymkowitz, F. Rousseau, A. Pastore, L. C. Serpell, and K. Broersen: J. Biol. Chem. 287 (2012) 5650.

20) K. Shinashi, T. Houkin, and H. Harada: Jpn. J. Appl. Phys. 51 (2012) 07GD05.

21) M. A. Margulis: Ultrasonics 23 (1985) 157.

22) Z. Xu and K. Yasuda: Jpn. J. Appl. Pnys. 50 (2011) 07HE07. 
Jpn. J. Appl. Phys.

Regular Paper

23) M. Cui, M. Jang, S. Lee, and J. Khim: Jpn. J. Appl. Phys. 51 (2012) 07GD13.

24) D. J. Watmough, M. B. Shiran, K. M. Quan, A. P. Sarvazyant, E. P. Khizhnyakt, and T. N. Pashovkint: Ultrasonics 30 (1992) 325.

25) M. Takahashi: J. Phys. Chem. B 109 (2005) 21858.

26) H. Naiki, K. Higuchi, M. Hosokawa, and T. Takeda, Anal. Biochem. 177 (1989) 244.

27) M. Biancalana, K. Makabe, A. Koide, and S. Koide, J. Mol. Biol. 385 (2009) 1052.

28) H. Ogi, Y. Fukunishi, T. Yanagida, H. Yagi, Y. Goto, M. Fukushima, K. Uesugi, and M. Hirao, Anal. Chem. 83 (2011) 4982.

29) Y. Yoshimura, Y. Lina, H. Yagia, Y. Lee, H. Kitayama, K. Sakurai, M. So, H. Ogi, H. Naiki, and Y. Goto: Proc. Natl. Acad. Sci. U. S. A 109 (2012) 14446.

30) H. Ogi, M. Fukushima, K. Uesugi, H. Yagi, Y. Goto, and M. Hirao, Biosens. Bioelectron. 40 (2013) 200.

31) J. S. Olsen, J. T. M. DiMaio, T. M. Doran, C. Brown, B. L. Nilsson, and S. Dewhurst: J. Biol. Chem. 287 (2012) 11842.

32) E. A. Neppiras: Phys. Rep. 61 (1980) 159.

33) D. L. Miller: Ultrasonics 19 (1981) 217.

34) B. Avvaru and A. B. Pandit: Ultrason. Sonochem. 16 (2009) 105.

35) N. Iefuji, R. Murai, M. Maruyama, Y. Takahashi, S. Sugiyama, H. Adachi, H. Matsumura, S. Murakami, T. Inoue, Y. Mori, Y. Koga, K. Takano, and S. Kanaya: J. Cryst. Growth 318 (2011) 741.

36) J. B. Keller and I. I. Kolonder: J. Appl. Phys. 27 (1956) 1152.

37) A. Prosperetti and A. Lezzi: J. Fluid Mech. 168 (1986) 457.

38) J. B. Keller and M. Miksis: J. Acoust. Soc. Am. 68 (1980) 628.

39) F. Rosenberger, S. B. Howard, J. W. Sowers, and T. A. Nyce: J. Cryst. Growth 129 (1993) 1.

40) A. K. Buell, A. Dhulesia, M. F. Mossuto, N. Cremades, J. R. Kumita, M. Dumoulin, M. E. Welland, T. P. J. Knowles, X. Salvatella, and C. M. Dobson: J. Am. Chem. Soc. 133 (2011) 7737. 
Jpn. J. Appl. Phys.

Regular Paper

\section{Figure Captions}

Fig. 1. Schematic of the experimental system.

Fig. 2. Waveforms (a) and corresponding FFT spectra (b) measured in microtubes when the $26 \mathrm{kHz}$ ultrasonic transducer is used. $\mathrm{f}_{0}$ denotes the fundamental frequency.

Fig. 3. Changes in the ThT level in $\mathrm{A} \beta_{1-40}$ solution at $\mathrm{pH} 4.6$ caused by UI at $26 \mathrm{kHz}$ (open squares), stirring at 1,200 rpm (open triangles), and incubation at room temperature (filled circles).

Fig. 4. Time courses of ThT level during UI at $26 \mathrm{kHz}$ for $\mathrm{A} \beta$ solutions of $\mathrm{pH}$ 7.4. Solid lines are fitted sigmoid functions. The dashed line indicates a decrease in ThT level due to breakage of the $\beta$-sheet structures.

Fig. 5. (Color online) Relationships between acoustic pressures of fundamental and harmonics waves with the ThT level at $5 \mathrm{~h}$ caused by UI. (a)-(c) are results with the $26 \mathrm{kHz}$ ultrasonic transducer and (d)-(f) are those with the $200 \mathrm{kHz}$ transducer. Black solid circles and red open circles denote results for solutions with $\mathrm{pH} 7.4$ and 4.6, respectively, and blue inverted triangles are results for the Ar-gas-saturated solution at $\mathrm{pH}$ 7.4. The linear correlation coefficients (CC) are shown.

Fig. 6. (Color online) AFM images $\left(5 \times 5 \mu \mathrm{m}^{2}\right)$ of $\mathrm{A} \beta$ aggregates produced by UI at $26 \mathrm{kHz}$ for $5 \mathrm{~h}$ for solutions with $\mathrm{pH} 4.6$ (a) and $\mathrm{pH} 7.4$ (b and c).

Fig. 7. (a) Calculated evolution of bubble radius at $26 \mathrm{kHz}$ (solid line) and $200 \mathrm{kHz}$ (dashed line) and (b) calculated temperature change near the bubble collapse at $26 \mathrm{kHz}$ in airsaturated water (dashed line) and Ar-saturated water (solid line). Inset shows enlarged temperature change near the peak for Ar-saturated water. Used parameters are as follows: $\gamma=1.4$ and 1.67 for air and Ar, respectively, $p_{\infty}=1.0 \mathrm{~atm}, P_{A}=1.6 \mathrm{~atm}, R_{0}=5.0 \mu \mathrm{m}$, $\rho_{\infty}=998.2 \mathrm{~kg} / \mathrm{m}^{3}, \sigma=7.275 \times 10^{-2} \mathrm{~N} / \mathrm{m}, \mu=1.002 \times 10^{-3} \mathrm{~Pa} \cdot \mathrm{s}$, and $c_{\infty}=1496 \mathrm{~m} / \mathrm{s}$. The initial temperature $T_{0}=25{ }^{\circ} \mathrm{C}$.

Fig. 8. Schematics of (a) phase diagram and (b) free energy diagram. (a) Phase diagram of A $\beta$ peptide will depend on concentration and temperature. Following Yoshimura et al., ${ }^{29)}$ we classified four representative regions: In region 1 (thermally stable region), native-state peptides exist. With an increase in concentration and temperature, their state moves to region 2 (metastable region), where the solution is in the supersaturated state, but no spontaneous nucleation occurs. A further increase in concentration and temperature causes the transition to region 3 (labile region), where spontaneous nucleation for fibril formation occurs. An excess increase in concentration and temperature will induce a transition to region 4 (glass region), where amorphous-like aggregates are precipitated. In our solutions, the peptide originally exists in region 2 , but ultrasonication will move the state to region 3 through local condensation and temperature increase. (b) Corresponding change in free energy landscape of $\mathrm{A} \beta$ peptide by UI. $\mathrm{A} \beta$ monomers produce nuclei 
Jpn. J. Appl. Phys.

Regular Paper

through intermediate aggregates. UI will lower the energies of intermediates through enhancement of supersaturation (red line). Because the energy barrier from intermediate states to the nucleus will remain unchanged, the energy level from monomer to nucleus is lowered. As the result, nucleation proceeds significantly, leading to fibrillation. 
Jpn. J. Appl. Phys.

Regular Paper

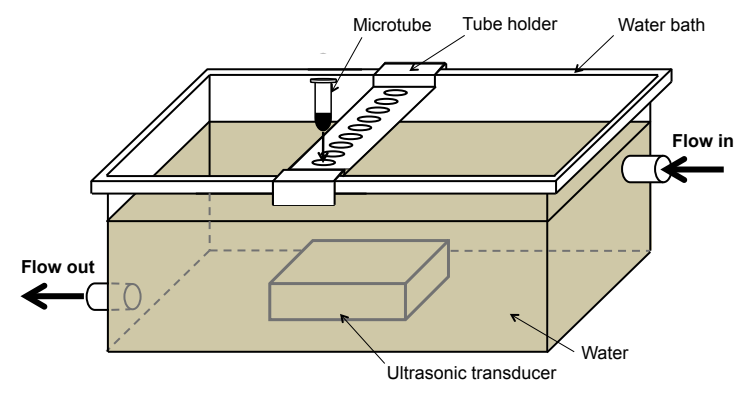

Fig. 1. 


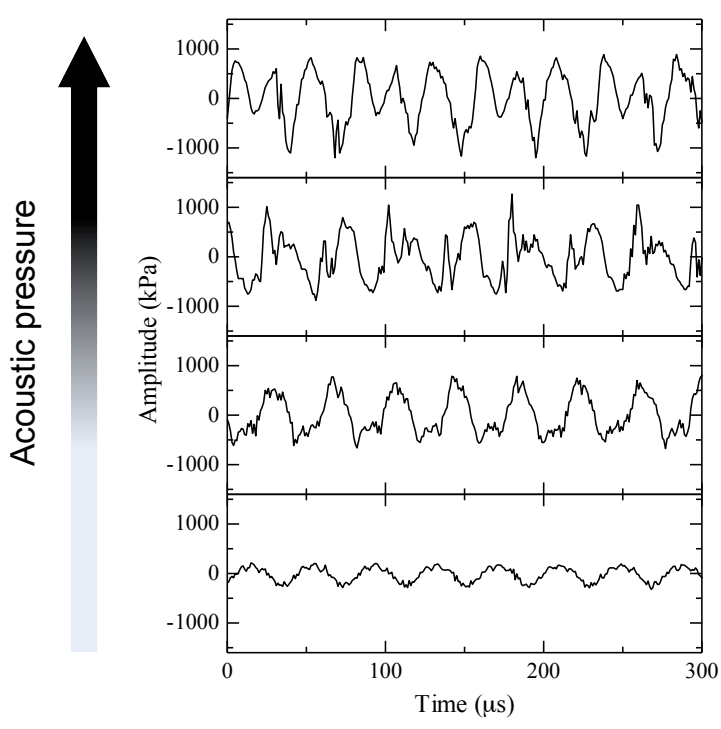

(a)

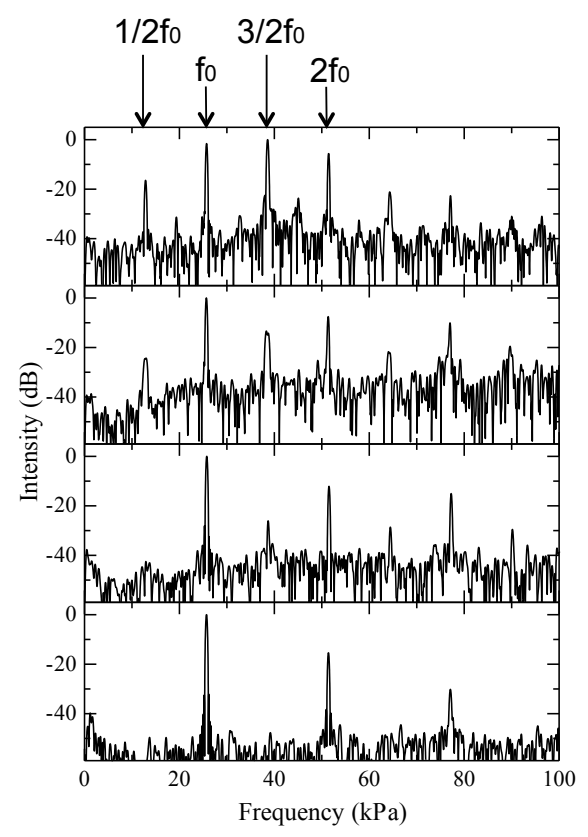

(b)

Fig. 2. 
Jpn. J. Appl. Phys.

Regular Paper

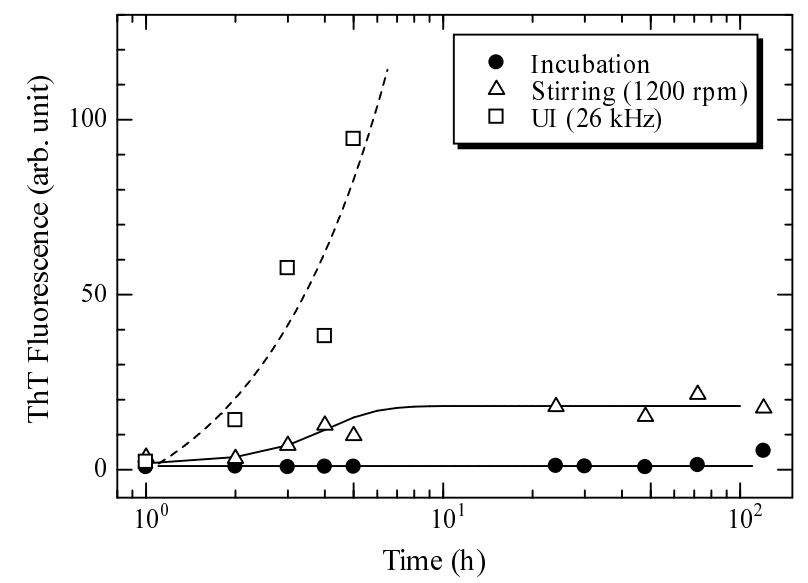

Fig. 3. 
Jpn. J. Appl. Phys.

Regular Paper

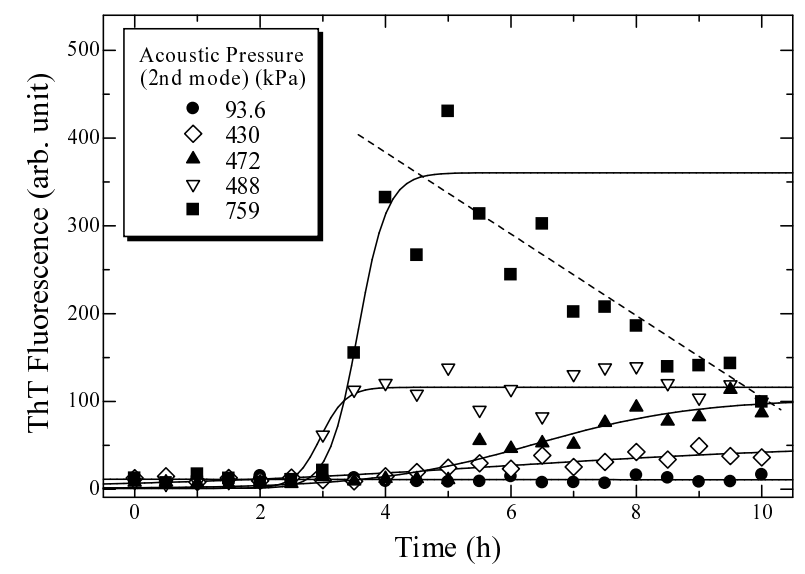

Fig. 4. 

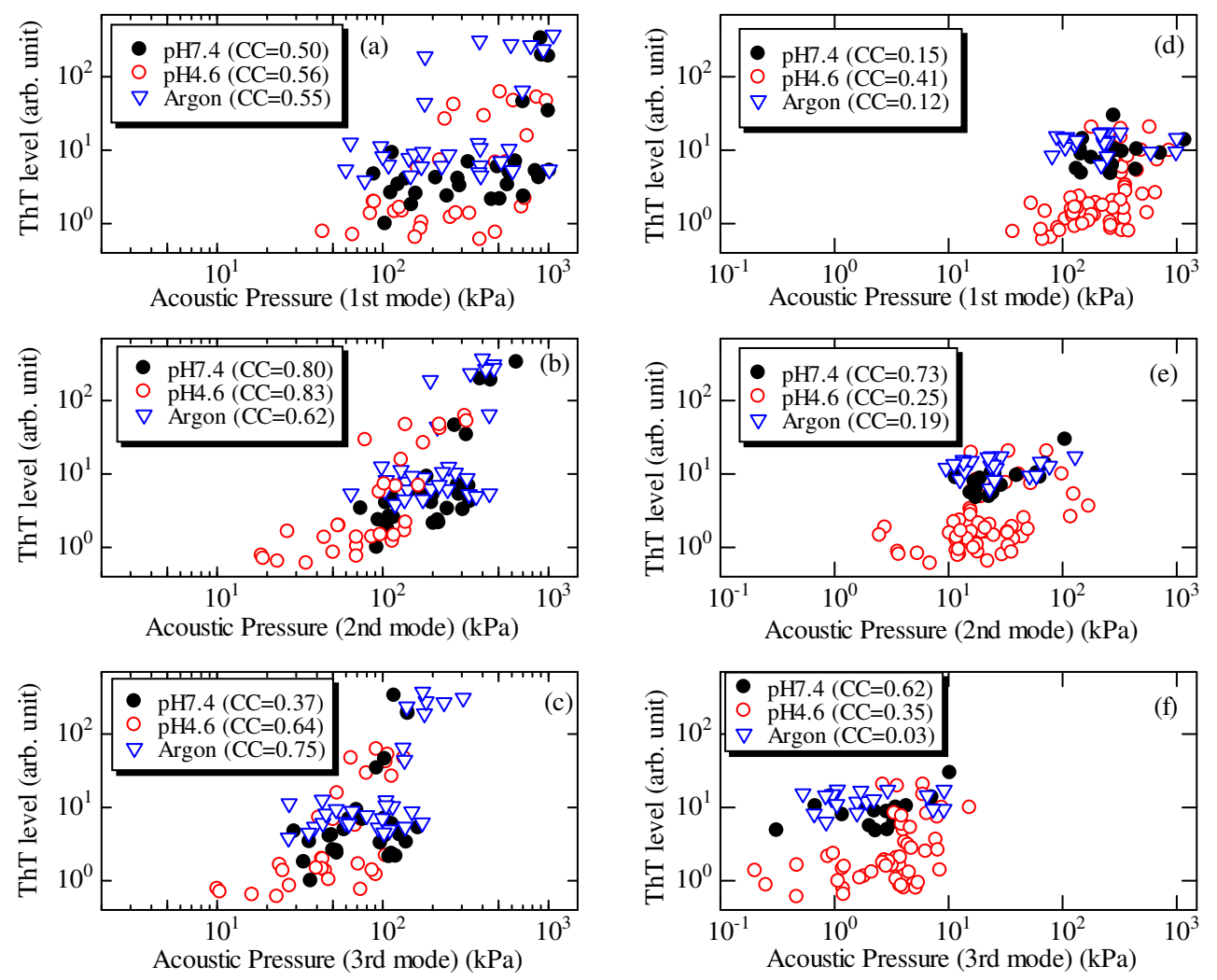

Fig. 5 . 
Jpn. J. Appl. Phys.

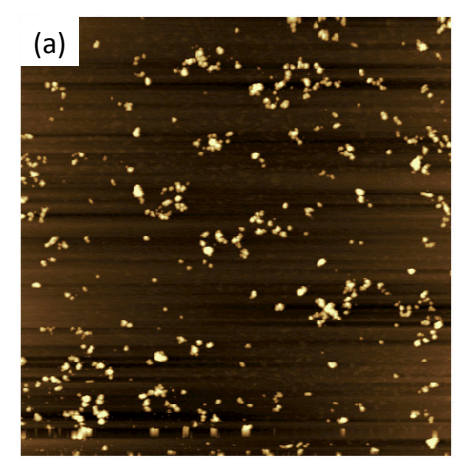

$1 \mu \mathrm{m}$

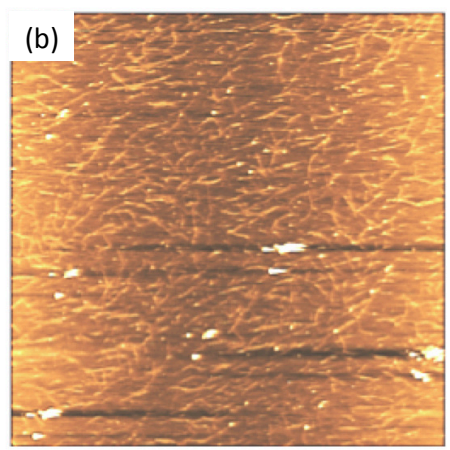

$1 \mu \mathrm{m}$
Regular Paper

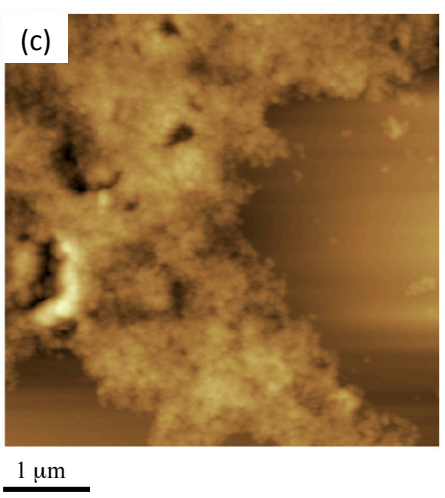

Fig. 6. 

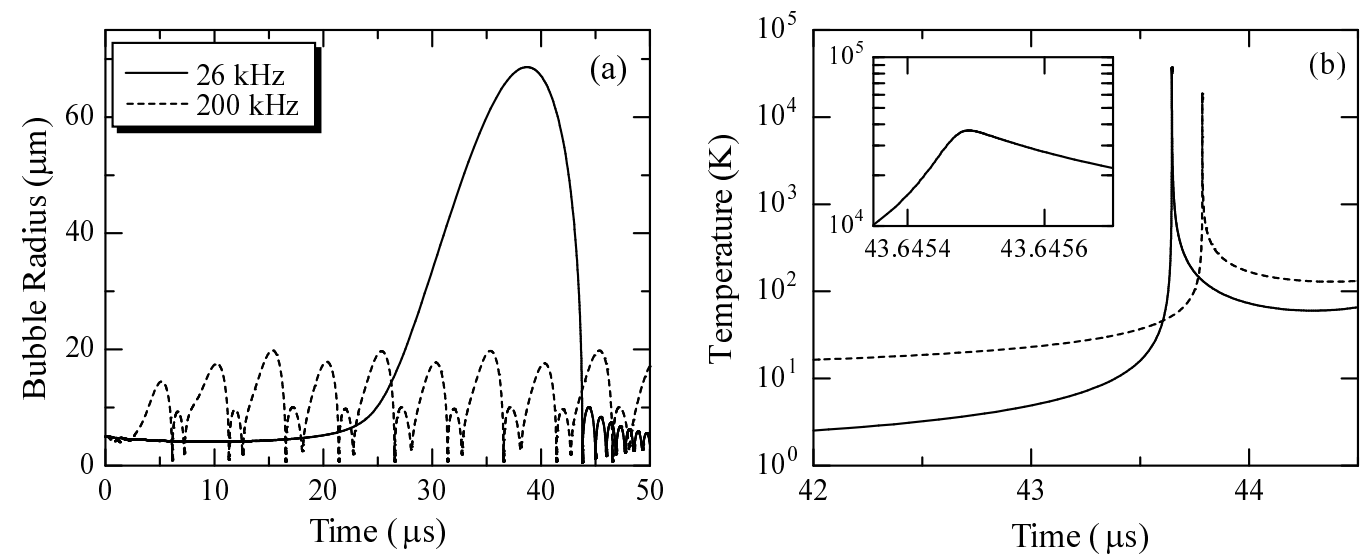

Fig. 7. 
Jpn. J. Appl. Phys.

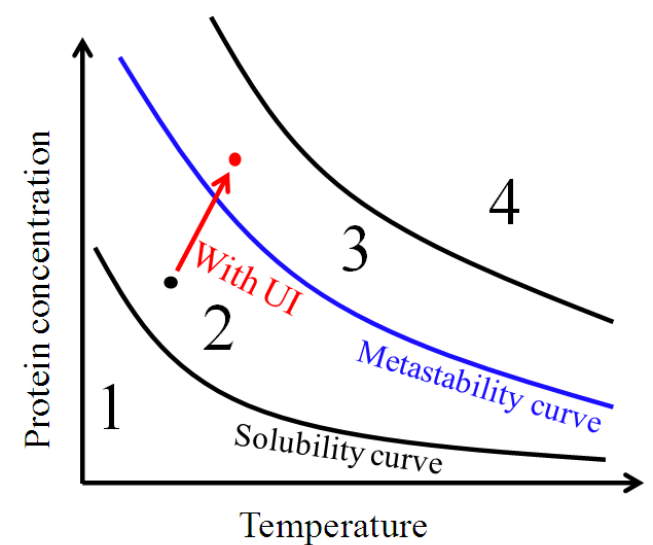

(a)

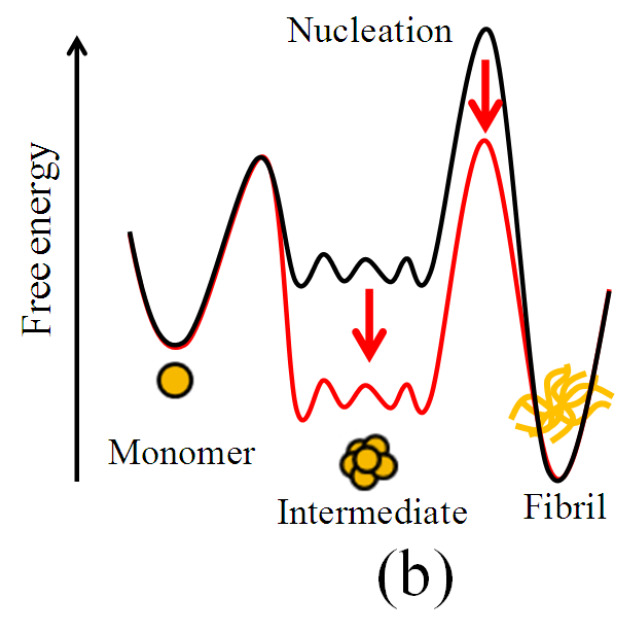

Fig. 8. 